\title{
The crypsis hypothesis explained: a reply to Jayasuriya et al. (2015)
}

\section{Torbjørn R. Paulsen ${ }^{1 \star}$, Louise Colville ${ }^{2}$, Matthew I. Daws ${ }^{4}$, Sigrunn Eliassen ${ }^{1}$, Göran Högstedt ${ }^{1}$, Ilse Kranner ${ }^{3}$, Ken Thompson ${ }^{5}$ and Vigdis Vandvik ${ }^{1}$}

${ }^{1}$ Department of Biology, University of Bergen, Postboks 7803, 5020 Bergen, Norway; ${ }^{2}$ Comparative Plant and Fungal Biology, Royal Botanic Gardens, Kew, Wakehurst Place, RH17 6TN, UK; ${ }^{3}$ Institute of Botany and Center for Molecular Biosciences Innsbruck, Sternwartestraße 15, University of Innsbruck, A-6020 Innsbruck, Austria; ${ }^{4}$ Alcoa of Australia Limited, PO Box 172, Pinjarra WA 6208, Australia; ${ }^{5}$ Department of Animal and Plant Sciences, University of Sheffield, Sheffield, S10 2TN, UK

(Received 8 June 2015; accepted without revision 6 July 2015; first published online 18 August 2015)

\begin{abstract}
In imbibing seeds, resumption of metabolism leads to the unavoidable release of volatile by-products that are perceived as cues by rodent seed predators. The crypsis hypothesis proposes that the primary function of a water-impermeable, hard seed coat is to reduce rodent seed predation by rendering seeds olfactorily cryptic. In an opinion paper, Jayasuriya et al. (2015) find the crypsis hypothesis unscientific and "not consistent with Darwin's theory of evolution by natural selection'. It is unfortunate that Jayasuriya et al. (2015) did not appreciate that the crypsis hypothesis offers an alternative explanation for the evolution of waterimpermeable seeds: released seed volatiles are cues used by rodents to locate seeds, and variation in seedcoat permeability leading to differences in seed volatile release represents the variable under selection. Furthermore, the sealing of water-impermeable seed coats imposes a cost of increased generation time and, therefore, dormancy-release mechanisms are expected to subsequently evolve in response to local environmental conditions. We also disagree with most other claims by Jayasuriya et al. (2015), who failed to appreciate how species with dimorphic seeds - one morph with permeable and the other with impermeable seed coats - benefit from rodent caching behaviour and population dynamics. We welcome this opportunity to clarify and elaborate on key features and the evolution of water-impermeable seed coats according to the crypsis hypothesis.
\end{abstract}

*Correspondence

Email: torbjorn.paulsen@bio.uib.no
Keywords: crypsis hypothesis for evolution of physical dormancy, physical dormancy, rodent, seed dispersal, seed predation, volatile compounds

\section{Introduction}

For at least a century, the literature examining the ecology and ecophysiology of water-impermeable hard seeds has been almost exclusively concerned with their dormancy function, as summarized by, for example, Baskin and Baskin (2014) and Jayasuriya et al. (2015). Inspired by the work on how rodents use smell to locate imbibed seeds by Vander Wall and others (e.g. Howard and Cole, 1967; Johnson and Jorgensen, 1981; Vander Wall, 1991, 1993a, b, 1995, 1998, 2000, 2010; Jorgensen, 2001; Vander Wall and Jenkins, 2003; Vander Wall et al., 2005), we revisited the dormancy hypothesis for the evolution of hard seeds, which led us to propose the crypsis hypothesis as an alternative explanation for the evolution of hardseededness, suggesting that the primary function of waterimpermeable hard seeds is their ability to reduce seed predation (Paulsen et al., 2013). We demonstrated that buried water-impermeable hard seeds are difficult to find for rodent seed predators that use volatiles as cues to locate seeds (Paulsen et al., 2013, and see also Vander Wall, 2003). With a mechanistic model, we also demonstrated that water-impermeable seed coats can reduce seed predation under a wide range of natural humidity conditions. Also, plants with rodent-dispersed seeds would benefit from producing dimorphic 'soft' (water permeable) and 'hard' (water impermeable) seeds at ratios where the anti-predator 
advantages of hard seeds are balanced by the dispersal benefits gained by producing some locatable soft seeds (Paulsen et al., 2014).

Water impermeability is not the only seed-coat trait that has been suggested to evolve as crypsis in response to seed predation. Porter (2013) suggested that avian seed predation selected for local seed colour camouflage in the legume Acmispon wrangelianus. Birds often have well-developed colour vision (Endler and Mielke, 2005) and have been shown to consume preferentially seeds most divergent from the background colour of substrates, under both experimental and field conditions (Nystrand and Granström, 1997; Saracino et al., 2004; Jones et al., 2006). Porter (2013) demonstrated that different lineages of $A$. wrangelianus, which grows on a variety of grey-green serpentine and brown non-serpentine soils, have seeds that closely match the colour of their native serpentine or non-serpentine soil type, and that this is the result of intraspecific genetic divergence. The lineages, even within the two soil types, produce seeds that more closely match the colour of the soil at their growth site than that of other sites.

In their opinion paper, Jayasuriya et al. (2015) state that they 'believe that some misconceptions about the evolution of PY have been incorporated into this hypothesis, and it does not consider other important selective pressures' (p. 127). In what appears to be their main conclusion, highlighted both in the abstract and concluding remarks, the crypsis hypothesis is considered unscientific in that the 'argument for evolution of the water-impermeable seed coat in response to predator pressure is not consistent with Darwin's (1859) theory of evolution by natural selection' (p. 133). It is unfortunate that Jayasuriya et al. (2015) fail to understand that the crypsis hypothesis incorporates the key mechanisms important for evolution by natural selection: predation is an extremely powerful selection pressure; seeds need to avoid being eaten before they even have an opportunity to germinate. Consequently, quite small variations in seed-coat thickness and permeability that restrict the ingress of water and escape of volatiles, could lead to the evolution of water-impermeable hard seeds.

From a dormancy perspective, physiological dormancy (PD) and physical dormancy (PY) could be viewed as two ways of dealing with the same problem: to prevent germination when conditions are unfavourable for seedling establishment. Arguably, PD does a better job because it is more flexible to changes in environmental conditions by constantly monitoring and responding to the surrounding environment. Moreover, PD is reversible, and can thus be reactivated to prevent a seed from germinating if conditions are no longer favourable, as opposed to PY where the dormancy breakage is irreversible (e.g. Baskin and Baskin, 2014; Willis et al., 2014). Due to its reversibility,
PD has more sophisticated dormancy functions than PY, but it has no function to prevent seed predation. On the other hand, PY 'camouflages' seeds from rodent predators but, once broken, most seeds germinate as soon as water is present, which makes it a questionable dormancy trait. Nevertheless, according to the dormancy hypothesis for the evolution of PY, the dormancy function is assumed to be the reason for the evolution of these seeds, as hard seeds evolved from a PD ancestor (Willis et al., 2014). Jayasuriya et al. (2015) argue that:

However, it seems that this is a general phenomenon that can be seen throughout evolution, i.e. there are several examples of more than one strategy performing the same function. Thus, just because seeds with PY cannot do anything better than those with PD does not invalidate the fact that PY plays a significant role in timing of germination to the correct period for seedlings to establish, i.e. it is another way of achieving the same outcome. (p. 129)

Yes, evolution can solve the same evolutionary problem in different ways in different populations, but that is not the case here as hard seeds evolved from and replaced PD. Having an impermeable seed coat does indeed regulate the timing of germination; since germination cannot take place until the seed coat is broken, it could hardly do otherwise. However, that does not necessarily imply that this was the main function on which natural selection acted and that water-impermeable hard seeds evolved for that purpose. In short, we argue that water impermeability provided an obvious selective advantage: protection from predation. Its advantages in terms of germination regulation are less obvious.

\section{Alternative hypothesis}

Jayasuriya et al. (2015) discuss the crypsis hypothesis as if it were a modified dormancy hypothesis. They claim that the variation explained is 'dormancy in our case' (p. 127). It is not. The crypsis hypothesis is an alternative hypothesis for the evolution of water-impermeable hard seeds. According to this hypothesis, the trait under selection is seed-coat permeability leading to differences in the seed volatiles ('smell') released as seeds imbibe and used by rodents as cues to locate seeds (Paulsen et al., 2013, p. 496; 2014, p. 1476).

The crypsis hypothesis explains the evolution of water-impermeable seeds and how rodent seed predation is expected to influence optimal hard/soft seed morph ratios in plants with water-impermeable seeds. The crypsis hypothesis also makes predictions about other seed traits associated with water-impermeable hard seeds, explaining general patterns observed in 
natural populations that would otherwise need ad hoc explanations (Paulsen et al., 2013, 2014). However, as with all general ecological explanations, exceptions to the rule are not only expected, but welcome, since they provide information on the limits of the general pattern.

\section{Evolution}

According to Jayasuriya et al. (2015):

The fundamental argument in the crypsis hypothesis, that PY evolved as an escape mechanism from predators, is not valid according to the evolutionary theory of Darwin. According to Darwin's hypothesis, variations (dormancy in our case) within a population occur randomly, i.e. there is no direct function of a variation at the time of its origin. (p. 127)

This is a misrepresentation of our hypothesis. The seed volatiles released differ between seeds (e.g. Hollander et al., 2012) giving variation both within and between species. Selection by rodents, assuming that variation in seed-coat permeability is heritable, is expected to result in directional selection towards seeds releasing less volatiles until we end up with water-impermeable olfactionally cryptic seeds (Vander Wall, 2003; Paulsen et al., 2013). Since these seeds are likely to have evolved from an ancestor with PD (Willis et al., 2014), the predator escape benefit of producing waterimpermeable seeds must exceed the cost of increased generation time presumably imposed on this new seed morph. Postponed germination is a by-product of the impermeable seed coat, not the function under selection, according to our hypothesis. The sealing of water-impermeable seed coats could thus be seen as a first evolutionary step, after which selection for release mechanisms could subsequently act (Paulsen et al., 2014).

\section{Release mechanisms}

Release mechanisms are expected to evolve, irrespective of the reason why water-impermeable seed coats evolved in the first place, as a way of reducing the cost of increased generation time in these seeds. As stated earlier:

For the seed to germinate, the seed coat must become water permeable at some point, and any structures synchronizing this to times or places where germination and seedling survival are heightened will reduce the cost of increased generation time in olfactionally cryptic hard seeds. (Paulsen et al., 2014, p. 1483)
To be absolutely clear: any structures or mutations in water-impermeable seeds giving rise to release mechanisms are expected to be quickly synchronized by natural selection with times and/or places where conditions are optimal for seedling establishment, because this would give the highest plant fitness. This is what we see. There is a huge variety of release mechanisms well adapted to local environmental conditions (Baskin and Baskin, 2014; Jayasuriya et al., 2015). The crypsis hypothesis treats the sealing and subsequent opening of these seeds as two distinct evolutionary steps, both of which arise from their own randomly occurring variation (in seed 'smell' and generation time, respectively) and selective advantage (reduced seed predation and shorter generation time, respectively). Therefore, the occurrence of release mechanisms per se does not support the assumption that dormancy is the primary driver for the evolution of water-impermeable seeds.

\section{Rodent behaviour}

Jayasuriya et al. (2015) appear to ignore the influence of rodent behaviour as an underlying mechanism for the crypsis hypothesis, and state categorically that:

However, even if we accept the hypothesis suggested by Paulsen et al. (2013), both hard seeds and soft seeds should be consumed similarly as soon as they are detected. If soft seeds are detected in higher numbers, then they will be consumed in higher numbers, and thus the selective pressure would have already eliminated the soft seeds. (pp. 129-130)

This is a deductive fallacy. Jayasuriya et al. (2015) assume that rodents will automatically eat located seeds. This is often not the case, because many rodents store seeds for later consumption.

This caching behaviour is hard to saturate, and rodents will harvest and cache far more seeds than they are able to eat (Chettleburgh, 1952; Vander Wall and Balda, 1977; Ligon, 1978; Darley-Hill and Johnson, 1981; Tomback, 1982; Vander Wall, 1988, 2002). From the plant's perspective, selection acts on plant fitness, not individual seed survival, and the presence of soft seeds in a mixed cache potentially increases plant fitness by increasing dispersal benefits (Paulsen et al., 2014). Rodents are thieving little creatures and will pilfer caches left by neighbours whenever they get the chance (e.g. Vander Wall and Jenkins, 2003). The presence of soft seeds can disclose the location of caches on a neighbour's territory allowing these to be pilfered. Furthermore, rodents have a very good spatial memory of where they have placed stored caches (Jacobs and Liman, 1991; Vander Wall, 1991, 2000; Jacobs, 1992) and they will therefore have no 
problem recovering caches containing only hard seeds that they have previously stored.

Jayasuriya et al. (2015) also assume that once their seed coat is breached hard seeds are exposed to predation in the same way as soft seeds. Jayasuriya et al. (2015) are mistaken again - they are not. Ignored hard seeds that imbibe and germinate in spring have a much higher chance of escaping rodent predation than soft seeds imbibing in the autumn, for at least four reasons. First, the number of rodents in a population often varies annually, with highest numbers in the autumn and lowest in spring, with winter mortality reported to be around $80 \%$ (e.g. Pucek et al., 1993). Second, there is seasonality in rodent caching behaviour, with highest caching activity in the autumn and declining cache recovery until late spring, when it seems to come to an end (Thompson and Thompson, 1980; Vander Wall, 1990). Third, the findings from the mixed seed experiments (Paulsen et al., 2013, fig. 2b and e, p. 499 and table S3 in the supporting information) demonstrated that water-impermeable hard seeds were left behind during cache harvest. These ignored seeds, individually scattered and buried during cache harvest, should be very hard for a small rodent spring population to re-locate by olfaction in the short timeframe between imbibition and germination (Reichman, 1979); and, fourth, the probability of successful germination and seedling establishment from ignored caches has been found to be high (e.g. Reichman, 1979; McAuliffe, 1990).

Our predictions rely on clearly outlined assumptions based on empirical data, as opposed to the misconceptions in the arguments put forward by Jayasuriya et al. (2015). The claims that 'If soft seeds are detected in higher numbers, then they will be consumed in higher numbers' (pp. 129-130) and that 'during the time of dormancy break to germination they [hard seeds] have the same risk of being detected by a predator as do soft seeds during the time from dispersal to germination' (p. 130) are not supported by rodent caching behaviour and population dynamics.

\section{Seed size}

Jayasuriya et al. (2015) asked for a definition of 'small seeds':

It is not clear how Paulsen et al. (2013) define 'small seeds', and we have to ask if they consider seeds of legumes (with PY), such as those of Trifolium campestre with a mass of $0.0004 \pm 0.0001 \mathrm{~g}$ (Baskin and Baskin, unpublished data), to be small or large. (p. 130)

This is another misrepresentation of our hypothesis. We argued that the absence of physical dormancy from 'lineages with predominantly small seeds' (Paulsen et al., 2013, p. 501) is consistent with our hypothesis.
Citing a single outlier among the Fabaceae with a moderately small seed is a red herring. The Fabaceae (the largest family with PY) is a generally large-seeded family.

Nevertheless, Hulme (1998) studied rodent removal rates of surface and buried seeds of, amongst others, Trifolium pratense, Trifolium dubium and Trifolium repens with seed masses of $0.59,1.92$ and $0.63 \mathrm{mg}$, respectively. Twenty-seven to $57 \%$ of offered surface seeds of all three species and $21-46 \%$ of buried $T$. pratense seeds were harvested, as were $36-48 \%$ of the surface seeds of Holcus lanatus, Poa annua and Poa pratensis with seed masses of $0.43,0.36$ and $0.37 \mathrm{mg}$, respectively, demonstrating that rodents will harvest seeds of these sizes. Therefore, we have no reason to believe that $T$. campestre seeds with a mass of about $0.4 \mathrm{mg}$ are too small to be harvested by rodents, and they are thus expected to benefit from being water impermeable in accordance with the crypsis hypothesis.

\section{Model interpretation}

In our 'model paper' (Paulsen et al., 2014) we describe how seed soil survival in different environments varies as a function of plant seed-morph strategy, i.e. the ratio of hard and soft seeds in dimorphic plants. Since plants naturally produce different proportions of hard and soft seeds (Morrison et al., 1992; Thanos et al., 1992; Meisert, 2002), we can predict under which environmental conditions selection would favour one strategy over another. We base our predictions on clearly outlined assumptions based on experimental findings and evolutionary modelling. Selection acts on the phenotype; in this case plant seed-morph strategy, and not on individual seeds per se as argued by Jayasuriya et al. (2015, pp. 129-130): 'If soft seeds are detected in higher numbers, then they will be consumed in higher numbers, and thus the selective pressure would have already eliminated the soft seeds'. If we assume that there is a higher germination success in seeds that are successfully dispersed from under the mother plant, we may expect plants to produce a mixture of hard and soft seeds (Paulsen et al., 2014). The proportion of soft seeds is also predicted to increase if there is a competitive advantage of early germination, shorter generation time, and we even make predictions about the distribution of hard and soft seeds given that there is a dormancy advantage to water-impermeable seeds. These are all testable predictions that can be refuted by empirical data.

\section{False claims}

Although we explicitly acknowledge that seed predation is not the only selective force affecting seed-coat 
morphology, Jayasuriya et al. (2015) claim that we did 'not consider other important selective pressures' (p. 127). On the contrary, our model explores the interaction between crypsis mechanisms and other factors that are 'not related to rodent dispersal or predation but accounts for other factors that may affect germination of the two seed morphs differently' (Paulsen et al., 2014, p. 1479). This gives us the possibility of examining the combined effect of several concomitant selective pressures without detailing each individual contribution (Paulsen et al., 2014, fig. 3, p. 1480 and fig. S1b).

Further, Jayasuriya et al. (2015) claim that 'If, as suggested by Paulsen et al. (2013), seed-eating predators are the only selection pressure operating on the kind of seed dormancy, PY should be dominant among the arid desert species of Geraniaceae' (p. 129). Again, we never claimed that rodents are the 'only selection pressure operating' on water-impermeable seeds in arid or any ecosystems. Jayasuriya et al. (2015) refer to a section where we discuss the hygroscopic nature of soft seeds and how difficult it is to remain olfactionally undetectable:

Even high relative humidity (95\%), assumed to produce dew and increase soil water vapour, was enough to make buried Jeffrey pine seeds olfactionally detectable for yellow pine chipmunks (Downs \& Vander Wall, 2009), and nightly dew formation (Jacobs et al., 1999) will make soft seeds detectable by olfaction on a daily basis, and thus an easy prey for foraging rodents. This could explain why hard seeds are surprisingly frequent in hot deserts (Baskin \& Baskin, 1998), where granivory by small rodents is very intense (Price \& Joyner, 1997), exerting very strong selection pressure on seeds to evolve methods of avoiding detection (Vander Wall, 2003). (Paulsen et al., 2013, p. 500)

Please note that we use the wording 'could explain' and 'surprisingly frequent'. This wording is chosen because it seems contradictory to the crypsis hypothesis that hard seeds should be present at all in arid systems, where seeds are not expected to need protection against moisture, and not as an argument that hard seeds should be very frequent in arid environments. However, since seeds are very hygroscopic and dew is enough to make seeds olfactionally detectable to rodents, many arid environments are probably not dry enough to protect seeds from rodents using volatiles as cue to locate seeds. Therefore, the claimed contradiction 'Paulsen et al. (2014) proposed an argument that is contradictory to that of Paulsen et al. (2013) regarding the high occurrence of PY seeds in dry environmental conditions' (Jayasuriya et al., 2015, p. 129) is not a contradiction at all.

Jayasuriya et al. (2015) also state that 'According to Paulsen et al. $(2013,2014)$, the only way to explain the presence of impermeable and permeable ('dimorphic') seeds in the same seed cohort is that water-permeable seeds are eaten by rodents, but the impermeable ones are taken (but not eaten) and cached' (p.129). We never claimed that soft seeds are eaten while hard seeds are cached, but wrote 'When the rodent finds a cache, it will eat a fraction of the seeds, recache some and neglect others' (Paulsen et al., 2014, p. 1476) and in fig. 1 (p. 1479) it is clear that predation rates on hard and soft seed morphs depend on the relative presence of each morph in a given cache.

Jayasuriya et al. (2015) also state that 'They do not mention sensitivity cycling in seeds with PY...' (p. 133). This is incorrect. We wrote 'Physical dormancy can be broken only once, although sensitivity to dormancy-breaking stimuli can cycle in some species' (Paulsen et al., 2013, p. 496). Sensitivity cycling (resulting in a cyclic germination pattern) in some seeds with PY, which had to evolve as a release mechanism after the evolution of a water-impermeable seed coat, does not change our conclusion that PD does a better job than hard seeds as a dormancy mechanism.

\section{Concluding remarks}

The crypsis hypothesis offers an explanation for the evolution of water-impermeable seeds, consistent with the widely supported view that rodents exert strong selective pressure, impacting on seed morphology and physiology. However, due to the variations between and within biomes, and the nature of general ecological patterns, we also expect to find examples that appear to contradict our hypothesis. Although olfactional crypsis is a probable and plausible explanation for the evolution of water-impermeable hard seeds, the crypsis hypothesis is 'young' and will need to develop further as empirical data to test the hypothesis become more abundant and available. The hypothesis is based on experimentally demonstrated mechanisms, and makes clear predictions that we hope will encourage rigorous empirical testing. This simple hypothesis, through the two evolutionary favourable steps of sealing (reduced seed predation) and re-opening the seed coat (reduced costs of increased generation time), explains why impermeability has evolved multiple times, as well as the diversity of locally adapted release mechanisms seen today.

\section{Acknowledgement}

Open access was funded by the University of Bergen. 


\section{Conflicts of interests}

None.

\section{References}

Baskin, C.C. and Baskin, J.M. (1998) Seeds. Ecology, biogeography, and evolution of dormancy and germination. San Diego, California, Academic Press.

Baskin, C.C. and Baskin, J.M. (2014) Seeds. Ecology, biogeography, and evolution of dormancy and germination (2nd edition). San Diego, Elsevier/Academic Press.

Chettleburgh, M.R. (1952) Observations on the collection and burial of acorns by jays in Hainault Forest. British Birds 45, 359-364.

Darley-Hill, S. and Johnson, W.C. (1981) Acorn dispersal by the Blue jay (Cyanocitta cristata). Oecologia 50, 231-232.

Darwin, C. (1859) On the origin of species by means of natural selection. London, John Murray.

Downs, C.J. and Vander Wall, S.B. (2009) High relative humidity increases pilfering success of yellow pine chipmunks. Journal of Mammalogy 90, 796-802.

Endler, J.A. and Mielke, P.W. (2005) Comparing entire colour patterns as birds see them. Biological Journal of the Linnean Society 86, 405-431.

Hollander, J.L., Vander Wall, S.B. and Longland, W.S. (2012) Olfactory detection of caches containing wildland versus cultivated seeds by granivorous rodents. Western North American Naturalist 72, 339-347.

Howard, W.E. and Cole, R.E. (1967) Olfaction in seed detection by deer mice. Journal of Mammalogy 48, 147-150.

Hulme, P.E. (1998) Post-dispersal seed predation and seed bank persistence. Seed Science Research 8, 513-519.

Jacobs, A.F.G., Heusinkveld, B.G. and Berkowicz, S.M. (1999) Dew deposition and drying in a desert system: a simple simulation model. Journal of Arid Environments 42, 211-222.

Jacobs, L.F. (1992) Memory for cache locations in Merriam's kangaroo rats. Animal Behaviour 43, 585-593.

Jacobs, L.F. and Liman, E.R. (1991) Grey squirrels remember the locations of buried nuts. Animal Behaviour 41, $103-110$.

Jayasuriya, K.M.G.G., Athugala, Y.S., Wijayasinghe, M.M., Baskin, J.M., Baskin, C.C. and Mahadevan, N. (2015) The crypsis hypothesis: a stenopic view of the selective factors in the evolution of physical dormancy in seeds. Seed Science Research 25, 127-137.

Johnson, T.K. and Jorgensen, C.D. (1981) Ability of desert rodents to find buried seeds. Journal of Range Management 34, 312-314.

Jones, K.A., Krebs, J.R. and Whittingham, M.J. (2006) Interaction between seed crypsis and habitat structure influences patch choice in a granivorous bird, the chaffinch Fringilla coelebs. Journal of Avian Biology 37, 413-418.

Jorgensen, E.E. (2001) Emission of volatile compounds by seeds under different environmental conditions. American Midland Naturalist 145, 419-422.

Ligon, J.D. (1978) Reproductive interdependence of Piñon jays and Piñon pines. Ecological Monographs 48, 111-126.
McAuliffe, J.R. (1990) Paloverdes, pocket mice, and bruchid beetles: interrelationships of seeds, dispersers, and seed predators. The Southwestern Naturalist 35, 329-337.

Meisert, A. (2002) Physical dormancy in Geraniaceae seeds. Seed Science Research 12, 121-128.

Morrison, D.A., Auld, T.D., Rish, S., Porter, C. and McClay, K. (1992) Patterns of testa-imposed seed dormancy in native Australian legumes. Annals of Botany 70, 157-163.

Nystrand, O. and Granström, A. (1997) Post-dispersal predation on Pinus sylvestris seeds by Fringilla spp.: ground substrate affects selection for seed color. Oecologia 110, 353-359.

Paulsen, T.R., Colville, L., Kranner, I., Daws, M.I., Högstedt, G., Vandvik, V. and Thompson, K. (2013) Physical dormancy in seeds: a game of hide and seek? New Phytologist 198, 496-503.

Paulsen, T.R., Högstedt, G., Thompson, K., Vandvik, V. and Eliassen, S. (2014) Conditions favouring hard seededness as a dispersal and predator escape strategy. Journal of Ecology 102, 1475-1484.

Porter, S.S. (2013) Adaptive divergence in seed color camouflage in contrasting soil environments. New Phytologist 197, 1311-1320.

Price, M.V. and Joyner, J.W. (1997) What resources are available to desert granivores: seed rain or soil seed bank? Ecology 78, 764-773.

Pucek, Z., Jedrzejewski, W., Jedrzejewska, B. and Pucek, M. (1993) Rodent population dynamics in a primeval deciduous forest (Białowieża National Park) in relation to weather, seed crop, and predation. Acta Theriologica 38, 199-232.

Reichman, O.J. (1979) Desert granivore foraging and its impact on seed densities and distributions. Ecology 60, $1086-1092$.

Saracino, A., D'Alessandro, C.M. and Borghetti, M. (2004) Seed colour and post-fire bird predation in a Mediterranean pine forest. Acta Oecologica 26, 191-196.

Thanos, C.A., Kyriacos, G., Kadis, C. and Pantazi, C. (1992) Cistaceae: a plant family with hard seeds. Israel Journal of Botany 41, 251-263.

Thompson, D.C. and Thompson, P.S. (1980) Food habits and caching behavior of urban grey squirrels. Canadian Journal of Zoology 58, 701-710.

Tomback, D.F. (1982) Dispersal of whitebark pine seeds by Clark's nutcracker: a mutualism hypothesis. Journal of Animal Ecology 51, 451-467.

Vander Wall, S.B. (1988) Foraging of Clark's nutcrackers on rapidly changing pine seed resources. The Condor 90, 621-631.

Vander Wall, S.B. (1990) Food hoarding in animals. Chicago, University of Chicago Press.

Vander Wall, S.B. (1991) Mechanisms of cache recovery by yellow pine chipmunks. Animal Behaviour 41, 851-863.

Vander Wall, S.B. (1993a) A model of caching depth: implications for scatter hoarders and plant dispersal. The American Naturalist 141, 217-232.

Vander Wall, S.B. (1993b) Seed water content and the vulnerability of buried seeds to foraging rodents. American Midland Naturalist 129, 272-281.

Vander Wall, S.B. (1995) Influence of substrate water on the ability of rodents to find buried seeds. Journal of Mammalogy 76, 851-856. 
Vander Wall, S.B. (1998) Foraging success of granivorous rodents: effects of variation in seed and soil water on olfaction. Ecology 79, 233-241.

Vander Wall, S.B. (2000) The influence of environmental conditions on cache recovery and cache pilferage by yellow pine chipmunks (Tamias amoenus) and deer mice (Peromyscus maniculatus). Behavioral Ecology 11, 544-549.

Vander Wall, S.B. (2002) Masting in animal-dispersed pines facilitates seed dispersal. Ecology 83, 3508-3516.

Vander Wall, S.B. (2003) How rodents smell buried seeds: a model based on the behavior of pesticides in soil. Journal of Mammalogy 84, 1089-1099.

Vander Wall, S.B. (2010) How plants manipulate the scatter-hoarding behaviour of seed-dispersing animals. Philosophical Transactions of the Royal Society B-Biological Sciences 365, 989-997.
Vander Wall, S.B. and Balda, R.P. (1977) Coadaptations of the Clark's nutcracker and the Pinon pine for efficient seed harvest and dispersal. Ecological Monographs 47, 89-111.

Vander Wall, S.B. and Jenkins, S.H. (2003) Reciprocal pilferage and the evolution of food-hoarding behavior. Behavioral Ecology 14, 656-667.

Vander Wall, S.B., Kuhn, K.M. and Beck, M.J. (2005) Seed removal, seed predation, and secondary dispersal. Ecology 86, 801-806.

Willis, C.G., Baskin, C.C., Baskin, J.M., Auld, J.R., Venable, D.L., Cavender-Bares, J., Donohue, K., de Casas, R.R. and The, N.G.W.G. (2014) The evolution of seed dormancy: environmental cues, evolutionary hubs, and diversification of the seed plants. New Phytologist 203, 300-309. 